\title{
Cooperation between the transcription factors p63 and IRF6 is essential to prevent cleft palate in mice
}

Helen A. Thomason, ${ }^{1}$ Huiqing Zhou, ${ }^{2}$ Evelyn N. Kouwenhoven, ${ }^{2}$ Gian-Paolo Dotto, ${ }^{3,4}$ Gaia Restivo, ${ }^{3}$ Bach-Cuc Nguyen, ${ }^{4}$ Hayley Little, ${ }^{5}$ Michael J. Dixon, ${ }^{1,5}$ Hans van Bokhoven, ${ }^{2}$ and Jill Dixon ${ }^{1}$

${ }^{1}$ Faculty of Medical and Human Sciences, Manchester Academic Health Sciences Centre, University of Manchester, Manchester, United Kingdom. 2Department of Human Genetics, Nijmegen Centre for Molecular Life Sciences, Radboud University Nijmegen Medical Centrum, Nijmegen, The Netherlands. ${ }^{3}$ Department of Biochemistry, University of Lausanne, Epalinges, Switzerland. ${ }^{4}$ Cutaneous Biology Research Center, Massachusetts General Hospital, Charlestown, Massachusetts, USA. ${ }^{5}$ Faculty of Life Sciences, University of Manchester, Manchester, United Kingdom.

\begin{abstract}
Cleft palate is a common congenital disorder that affects up to 1 in 2,500 live human births and results in considerable morbidity to affected individuals and their families. The etiology of cleft palate is complex, with both genetic and environmental factors implicated. Mutations in the transcription factor-encoding genes $p 63$ and interferon regulatory factor 6 (IRF6) have individually been identified as causes of cleft palate; however, a relationship between the key transcription factors p63 and IRF6 has not been determined. Here, we used both mouse models and human primary keratinocytes from patients with cleft palate to demonstrate that IRF6 and $p 63$ interact epistatically during development of the secondary palate. Mice simultaneously carrying a heterozygous deletion of $p 63$ and the Irf6 knockin mutation R84C, which causes cleft palate in humans, displayed ectodermal abnormalities that led to cleft palate. Furthermore, we showed that p63 transactivated IRF 6 by binding to an upstream enhancer element; genetic variation within this enhancer element is associated with increased susceptibility to cleft lip. Our findings therefore identify p63 as a key regulatory molecule during palate development and provide a mechanism for the cooperative role of p63 and IRF6 in orofacial development in mice and humans.
\end{abstract}

\section{Introduction}

Development of the secondary palate involves a complex series of integrated events that are frequently disturbed, resulting in the congenital malformation cleft palate. With an estimated incidence of 1 in 2,500 live births, depending on geographic origin, racial, and ethnic variation, and socioeconomic status $(1,2)$, cleft palate results in considerable morbidity to affected families, as individuals who exhibit this condition may experience problems with eating, speaking, and hearing that can be corrected to varying degrees by surgery, dental treatment, speech therapy, and psychosocial intervention $(3,4)$. The frequent occurrence and significant healthcare burden imposed by cleft palate emphasize the need to identify the molecular and cellular interactions that lead to facial clefting, with the ultimate aim of improving diagnosis, treatment, counseling, and care for affected individuals and their relatives.

In approximately $50 \%$ of cases, cleft palate occurs as an isolated entity; the remainder arise as part of a syndrome in which structures other than the palate are affected (5). The genetic basis of nonsyndromic clefting is complex, as variations in numerous genes, together with environmental factors, are known to play a role in its etiology (3-5). Recent advances in delineating the molecular mechanisms underlying cleft palate have therefore resulted largely from analysis of syndromic forms of cleft palate; for example, mutations in the p53 family member p63 and in interferon regulatory factor 6 (IRF6) underlie congenital malformation syn-

Authorship note: Helen A. Thomason and Huiqing Zhou contributed equally to this work.

Conflict of interest: The authors have declared that no conflict of interest exists. Citation for this article: J Clin Invest. 2010;120(5):1561-1569. doi:10.1172/JCI40266. dromes that display cleft palate as part of the phenotype (6-9). The combination of genetic studies in humans and analysis of targeted mutations in mice has identified several factors that play key roles during palate development $(10,11)$.

The IRF6 gene encodes a transcription factor characterized by a highly conserved DNA-binding domain in addition to a less well-conserved protein interaction domain (12). Mutations in IRF6 underlie Van der Woude syndrome (VWS) and popliteal pterygium syndrome (PPS), which are autosomal-dominant disorders characterized by varying combinations of cleft lip, cleft palate, lower lip pits, and dental, ectodermal, and genital anomalies (8). Importantly, genetic variants in and around IRF6 confer a significant attributable risk for nonsyndromic cleft lip (13). Gene targeting of the Irf6 locus has resulted in 2 mouse models: a knockin of the most common mutation found in PPS patients, R84C, which expresses a mutant Irf6 protein (14), and a complete loss-of-function allele (15). Although the function of the R84C mutation is still largely unknown, a recent study has demonstrated that this mutation results in loss of DNA binding (16). In both cases, homozygous mice exhibit a hyperproliferative epidermis that fails to undergo terminal differentiation and leads to severe intraoral epithelial adhesions $(14,15)$.

The p63 gene encodes at least 6 protein variants as the result of use of 2 different transcription start sites and alternative splicing. Different promoters give rise to 2 alternative $\mathrm{N}$-termini: transactivation sequence (TA) isoforms, which contain a TA similar to that in $\mathrm{p} 53$, and $\Delta \mathrm{N}$, isoforms which contain a shorter activation domain, TA2 (17). Alternative splicing toward the carboxy terminus generates 3 subtypes, $\alpha, \beta$, and $\gamma$ (18). All isoforms contain DNA-binding and isomerization domains, but vary in their ability to activate or repress their target genes $(19,20) . \Delta \mathrm{Np} 63 \alpha$ is the 
WT
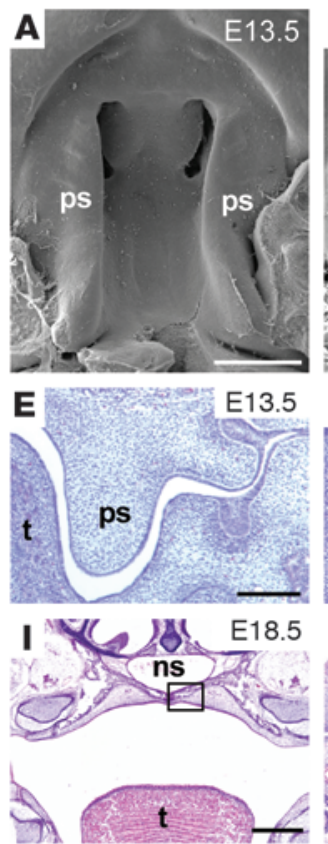

$p 63^{*-1} 1166^{* / 1844 C}$
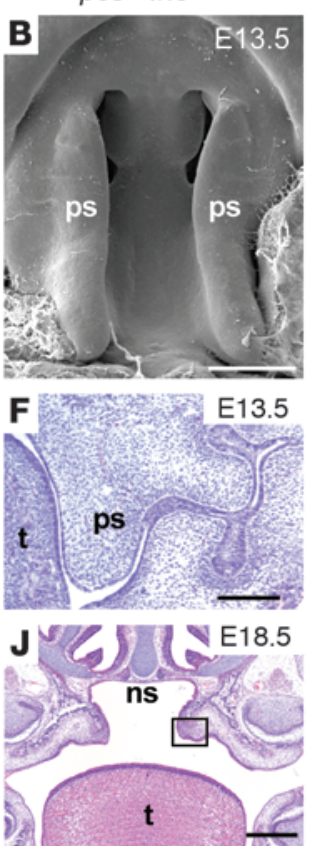
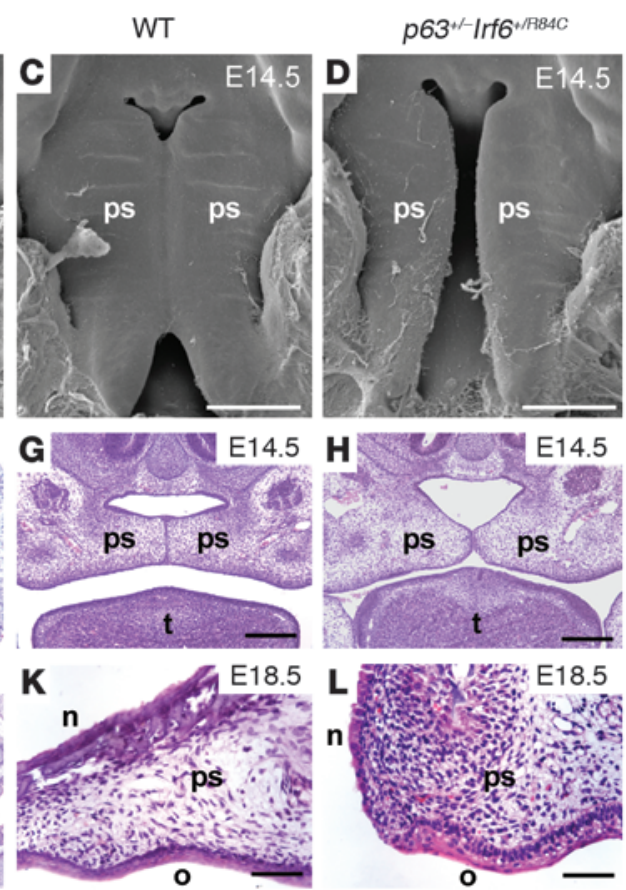

Figure 1

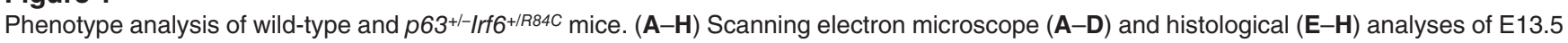

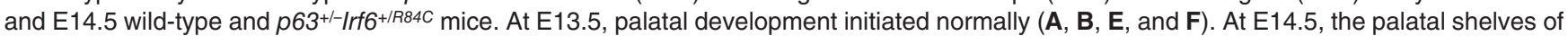

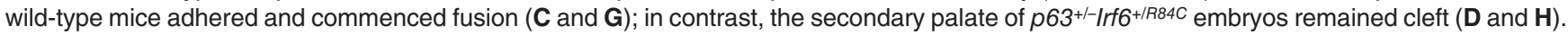

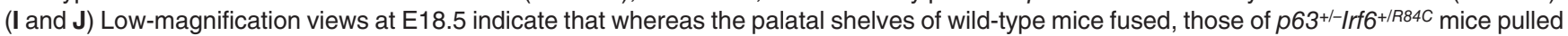
apart, leaving a large oronasal cavity. (K and $\mathbf{L}$ ) Higher-magnification views of the boxed regions in I and $\mathbf{J}$, respectively, indicate that the palatal epithelia of $p 63^{+/-I r f 6+/ R 84 C}$ embryos exhibited signs of differentiation with distinct nasal and oral characteristics. ps, palatal shelf; $t$, tongue; ns, nasal septum; n, nasal epithelium; o, oral epithelium. Scale bars: $500 \mu \mathrm{m}$ (A-D); $200 \mu \mathrm{m}$ (E-H); $400 \mu \mathrm{m}$ (I and J); $50 \mu \mathrm{m}$ (K and L).

major isoform expressed in basal epithelial cells and is essential for epidermal and palatal development $(17,21,22)$. Heterozygous mutations in $p 63$ underlie 7 autosomal-dominant developmental disorders that are characterized by varying combinations of cleft lip, cleft palate, ectodermal dysplasia, and limb abnormalities (7). To date, 2 mouse models of $p 63$ have been reported, one a loss-offunction allele (23) and another recently found to express $\mathrm{p} 63 \gamma$ isoforms $(24,25)$. Both mouse models exhibit a similar phenotype consisting of severe limb abnormalities, a thin and undifferentiated epidermis, and lack of epidermal derivatives $(23,24)$.

Despite the established roles of IRF6 and p63 in orofacial development, the molecular pathways in which they function during development of the lip and palate are poorly characterized. In the current study, we demonstrate that Irf6 and $p 63$ interact epistatically. Mice heterozygous for the p63 loss-of-function allele and for the Irf6 knockin mutation R84C exhibited cleft palate as a result of ectodermal abnormalities that occurred during palate development. To dissect the mechanism underlying this genetic interaction, we used a combination of chromatin immunoprecipitation (ChIP) and expression analyses to show that IRF6 was a direct target gene of $\mathrm{p} 63$ and that p63 activated IRF6 transcription through an enhancer element, variation within which increased susceptibility to cleft lip.

\section{Results}

Epistatic interaction between Irf6 and $p 63$ in palatal development. In light of the striking phenotypic overlap exhibited by syndromes resulting from mutations in IRF6 and p63, we investigated the poten- tial interaction between $p 63$ and Irf6 using an epistatic approach in which we intercrossed $p 63^{+/-}$and $\operatorname{Irf6} 6^{+/ R 84 C}$ mice. We examined 17 litters of $p 63^{+/-} I r f 6^{+/ R 84 C}$ mice between the ages of E14.5 and P0 $(N=145)$. Whereas mice heterozygous for the mutant $p 63$ allele $n=27)$ or a mutant Irf6 allele alone $(n=38)$ appeared grossly normal and comparable to their wild-type littermates $(n=45)$, approximately $89 \%$ of compound heterozygous $p 63^{+/-} \operatorname{Irf6} 6^{+/ R 84 C}$ embryos $(n=35)$ exhibited a cleft of the secondary palate. Scanning electron microscopy and histological analysis of a developmental series of embryos indicated that the initial stages of palatal development appeared normal. The palatal shelves of $p 63^{+/-} \operatorname{Irf6} 6^{+/ R 84 C}$ embryos grew vertically down the sides of the tongue and elevated at the expected developmental stage (Figure $1, \mathrm{~A}-\mathrm{C}$ and $\mathrm{E}-\mathrm{G}$ ); however,

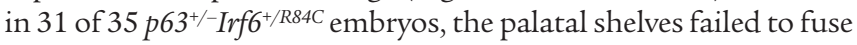
(Figure 1, D and H). Examination of 5 litters at E14.5 (yielding 15

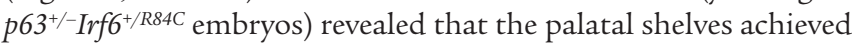
intimate contact (Figure 1H); nevertheless, with continued growth of the craniofacial complex, the $p 63^{+/-} \operatorname{Irf} 6^{+/ R 84 C}$ palatal shelves separated such that by E18.5, a large oronasal space was apparent (Figure 1J). Despite this observation, histological analysis indicated

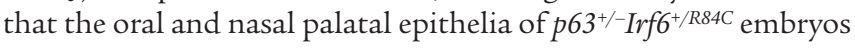
differentiated normally (Figure $1, \mathrm{~K}$ and L). Newborn $p 63^{+/-I r f 6^{+/ R 84 C}}$ mice exhibited gasping behavior and died shortly after birth. Histological and immunofluorescence analyses indicated that the epidermis of E18.5 $\mathrm{p} 63^{+/-} \mathrm{Irf6} \mathrm{C}^{+/ R 84 \mathrm{C}}$ embryos differentiated normally (Supplemental Figure 1; supplemental material available online with this article; doi:10.1172/JCI40266DS1). 

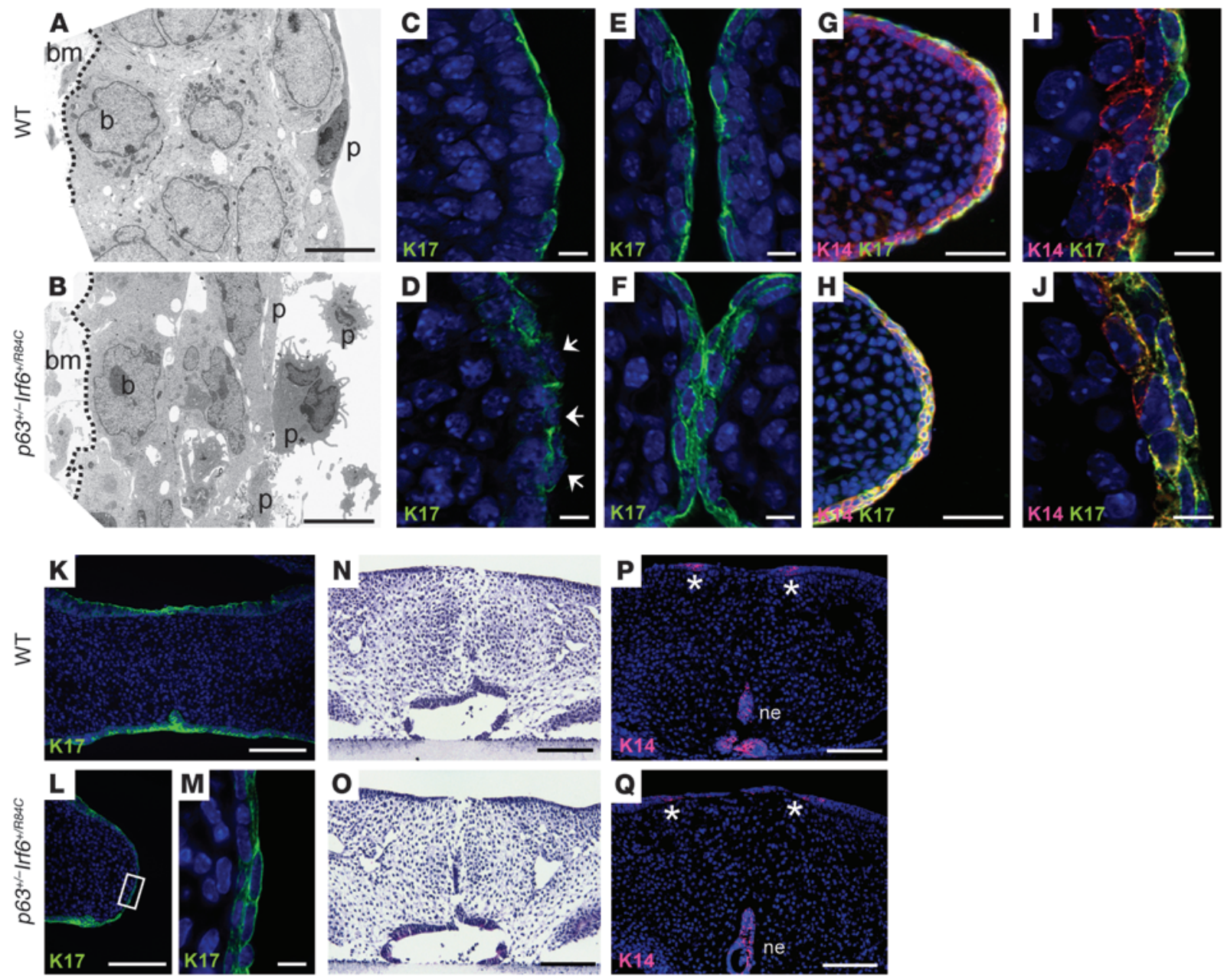

\section{Figure 2}

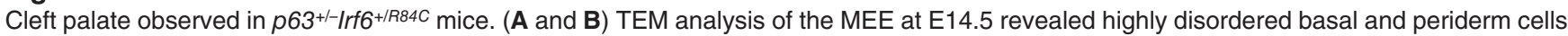
in p63+/Irf6+/R84C mice compared with their wild-type littermates. (C-J) Deconvolution analysis of the palatal shelves. (C and D) At E13.5, K17 appeared filamentous and incorrectly localized in the MEE (D, arrows). (E and F) At E14.5, whereas K17 expression was confined to the periderm of wild-type mice, K17 was expressed throughout the MEE in p63+-Irf6 $6^{+/ R 84 C}$ embryos. (G-J) K14 and K17 dual staining. (G and I) In wild-type

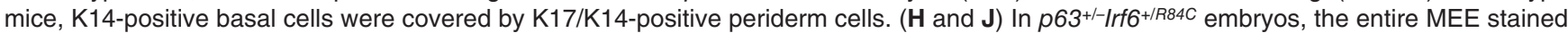
positively for both $\mathrm{K} 14$ and $\mathrm{K} 17$. (K-M) At E15.5, whereas the MEE of wild-type mice degenerated, K17-positive cells persisted over the MEE

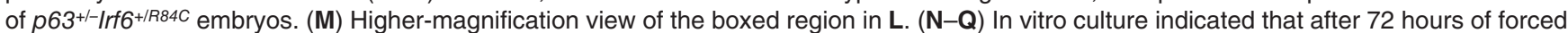

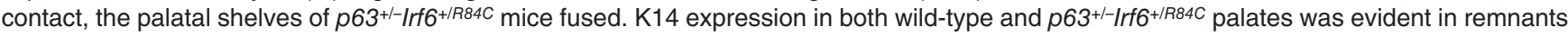
of the nasal and oral epithelia only ( $\mathbf{P}$ and $\mathbf{Q}$, asterisks). bm, basement membrane; $p$, periderm cell; $b$, basal cell; ne, nasal epithelium. Scale bars: $5 \mu \mathrm{m}$ (A and $\mathbf{B}) ; 10 \mu \mathrm{m}(\mathbf{C}-\mathbf{F}, \mathbf{I}, \mathbf{J}$, and $\mathbf{M}) ; 50 \mu \mathrm{m}$ (G and $\mathbf{H}) ; 100 \mu \mathrm{m}(\mathbf{K}, \mathbf{L}$, and $\mathbf{N}-\mathbf{Q})$.

To gain greater insight into the pathogenesis of the cleft palate observed in $p 63^{+/-} \operatorname{Irf6} 6^{+/ R 84 C}$ mice, we examined the morphology of the medial edge epithelium (MEE) from E13.5 to E15.5. As immunostaining for desmoplakin, $\alpha$ - and $\beta$-catenin, and E-cadherin indicated no gross differences in the distribution of these adhesion molecules between wild-type and $p 63^{+/-} \operatorname{Irf} 6^{+/ R 84 C}$ embryos (Supplemental Figure 2 and data not shown), we examined the palatal epithelia in greater detail using transmission electron microscopy. At E14.5, the MEE of wild-type mice consisted of a 2- to 3-cell-thick layer of basal and intermediate cells containing well-rounded nuclei, covered by a surface layer of flattened periderm cells (Figure $2 \mathrm{~A})$. In contrast, the equivalent region in $p 63^{+-} \operatorname{Irf} 6^{+/ R 84 C}$ embryos contained basal and intermediate cells with irregular nuclei covered by morphologically abnormal periderm cells (Figure 2B). We therefore examined the periderm in greater detail using immunostaining for keratin 17 (K17). Deconvolution images from E13.5 $p 63^{+/-} \operatorname{Irf6^{+/R84C}}$ embryos indicated that $\mathrm{K} 17$ immunostaining appeared filamentous and incorrectly localized (Figure 2, C and D). At E14.5, immunostaining was not confined to the most superficial cells; rather, K17 appeared to be expressed throughout the epithelium (Figure 2, E and F). This observation was confirmed by deconvolution analysis of K17/K14 dual-stained sections. In wild-type mice, the basal cells were positive for K14 only, with a superficial layer of periderm cells positive for $\mathrm{K} 14$ and $\mathrm{K} 17$; in contrast, in $p 63^{+/-} \operatorname{Irf} 6^{+/ R 84 \mathrm{C}}$ embryos, the entire MEE stained positively for both (Figure 2, G-J). Analysis of E15.5 $p 63^{+/-} \mathrm{Irf6} \mathrm{G}^{+/ R 84 \mathrm{C}}$ embryos using $\mathrm{K} 17$ immunostaining revealed that abnormal periderm cells persisted over the medial edge after the palates had fused in the wild-type embryos (Figure 2, K-M). 

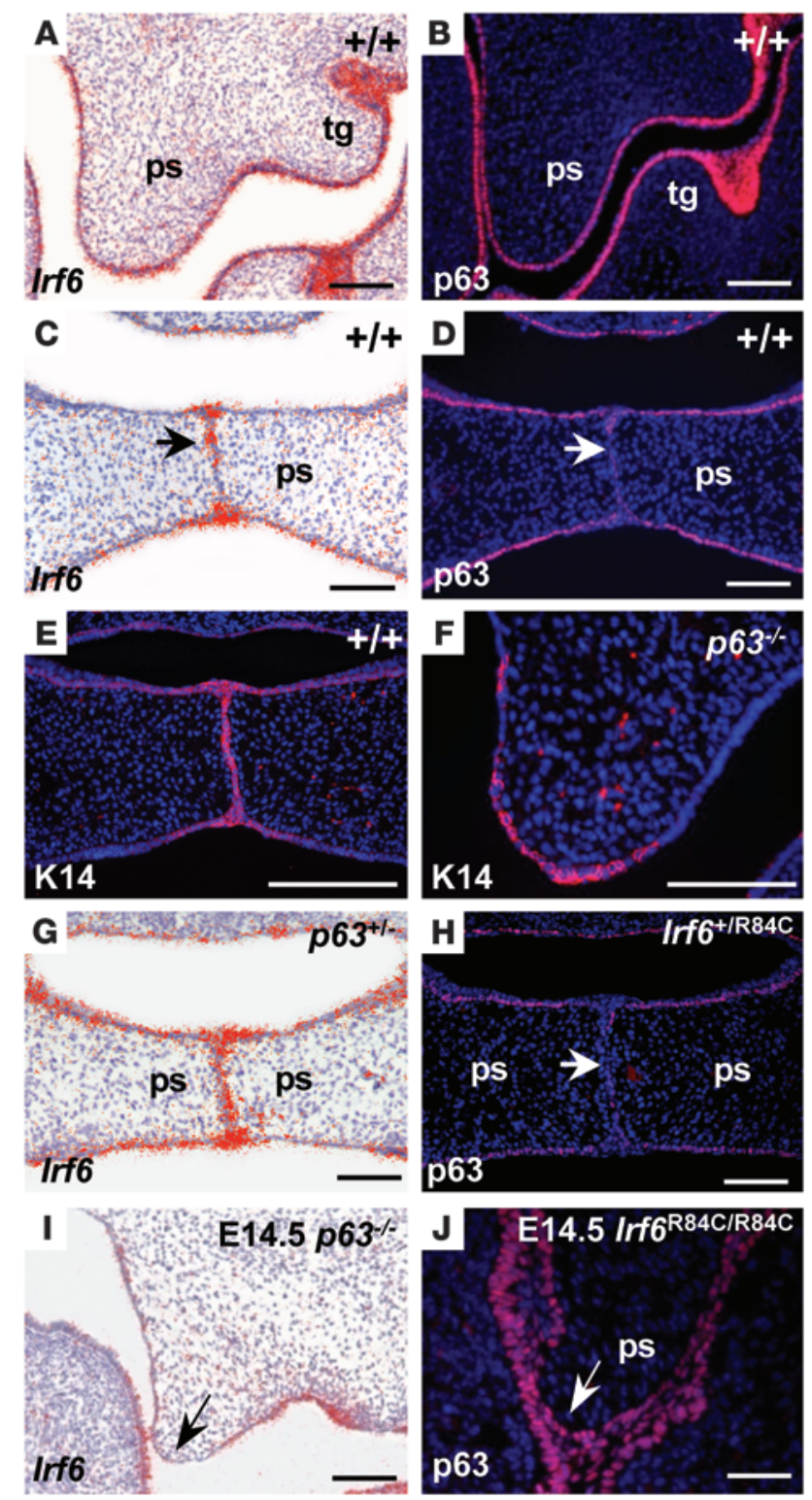

To assay whether the palatal shelves of $p 63^{+/-} \operatorname{Irf6} 6^{+/ R 84 C}$ embryos were competent to fuse, we used in vitro organ culture. Palatal shelves dissected from E13.5 wild-type and $p 63^{+/-} I r f 6^{+/ R 84 C}$ mice were placed in contact and cultured for 24,48 , or 72 hours. Histological analysis confirmed that after 72 hours, palatal fusion was essentially complete in embryos of both genotypes (Figure 2, N and $\mathrm{O}$ ). Immunofluorescence analysis confirmed that K14 was present only in patches of the oral and nasal epithelia (Figure 2, $\mathrm{P}$ and Q). K17 expression was undetectable, except in the tooth germs (data not shown).

As increased K17 immunoreactivity has previously been observed in hyperproliferative skin conditions (26), we performed cell pro-

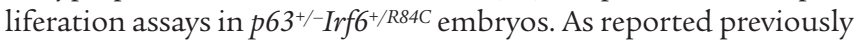
(27), the MEE of E14.5 wild-type mice had ceased proliferating (Supplemental Figure 2). In $p 63^{+/-} \operatorname{Irf6} 6^{+/ R 84 C}$ embryos, the abnormal cells of the MEE - both those of the basal layer and the abnormal periderm cells - were neither hyperproliferative nor undergoing abnormal cell death (Supplemental Figure 2).

\section{Figure 3}

Irf6 and p63 expression during palatal development. (A and B) At E13.5, Irf6 and p63 were expressed in similar domains in the epithelia of the oral cavity and in the tooth germs (tg) of wild-type mice. (C and D) At E14.5, Irf6 transcripts were strongly expressed in the midline seam and epithelial triangles (C, arrow); in contrast, p63 protein levels were downregulated in the MEE (D, arrow). (E and F) Immunofluorescence assays using $\mathrm{K} 14$ indicated that the palatal shelves of $p 63^{-/-}$mice, which exhibited a thin and fragile epithelium, were competent to express this protein. ( $\mathbf{G}$ and $\mathbf{H}$ ) Analysis of p63 and Irf6 in Irf6 $6^{+/ R 84 C}$ and $p 63^{+/-}$embryos, respectively. Expression was unchanged in the heterozygous animals. (I) In E14.5 $\mathrm{p63} \mathrm{3}^{-/-}$palatal shelves, Irf6 transcripts were downregulated in the MEE (arrow). (J) p63 expression was maintained throughout the MEE of E14.5 Irf6 ${ }^{R 84 C / R 84 C}$ palatal shelves (arrow). Scale bars: $100 \mu \mathrm{m}$.

IRF6 expression is dependent upon functional p63. Having established a genetic interaction between Irf6 and $p 63$, we next examined the expression of these molecules during development of the secondary palate in wild-type mice, in which the palatal shelves fuse, and in $p 63^{-/-}$and $\operatorname{Irf} 6^{R 84 C / R 84 C}$ embryos, both of which display cleft palate $(14,15,22,23)$. In E13.5 wild-type mice, p63 and Irf6 were expressed in similar domains in the oral epithelia and the developing tooth germs (Figure 3, A and B). Interestingly, at E14.5, although Irf6 expression was still detected in the midline epithelial seam, p63 expression was downregulated in this region (Figure 3D). To further characterize the interaction between these transcription factors, we examined their expression patterns in the corresponding mutant mice; however, as $p 63^{-/-}$epithelia are hypomorphic and slough off due to aberrant differentiation $(23,24)$, we first confirmed that $p 63^{-/-}$palatal epithelia were competent to express K14 (Figure 3, E and F). In $p 63^{+/-}$and Irf $6^{+/ R 84 C}$ mice, expression of Irf6 and p63, respectively, appeared unchanged from that observed in wild-type littermates (Figure 3, G and H); however, in $p 63^{-/-}$mice, Irf6 was downregulated in the epithelia at the tips of palatal shelves on E13.5 (data not shown) and E14.5 (Figure 3I). Strikingly, in contrast to its expression in wild-type and $\operatorname{Irf6} 6^{+/ R 84 C}$ mice, p63 was not downregulated in the presumptive MEE in Irf6 ${ }^{R 84 C / R 84 C}$ mice (Figure 3J). Real-time quantitative PCR (qPCR) assays, performed on RNA extracted from palatal shelves dissected from E13.5 mice, confirmed that Irf6 expression in $p 63^{-1-}$ embryos was downregulated significantly compared with $p 63^{+/-}$and wildtype littermates $(P=0.01$ for both comparisons, Mann-Whitney $\mathrm{U}$ test; Figure $4 \mathrm{~A})$. As isolated palatal epithelial cells have not been cultured successfully to our knowledge, we investigated the relationship between $p 63$ and Irf6 using mouse primary keratinocytes. Using siRNA, we knocked down $p 63$ (Figure 4B) and found a statistically significant decrease in the level of $\operatorname{Irf} 6$ transcript $(P=0.05$, Mann-Whitney U test; Figure 4C). Western blot analysis also indicated decreased protein levels (Figure 4D). These data suggested that p63 may regulate Irf6 expression.

To extend the observations obtained in mice, we assessed whether IRF6 expression is affected in a human disease model. We established human primary keratinocyte cell lines from 3 patients with ectrodactyly-ectodermal dysplasia-clefting (EEC) syndrome, who exhibit missense mutations in the DNA-binding domain of p63 (7). These patients have phenotypes typical of EEC syndrome, including cleft lip and palate. Real-time qPCR analysis of the R204W, R279H, and R304W mutant cell lines after 48 hours of differentiation indicated that p63 transcript levels were similar to 

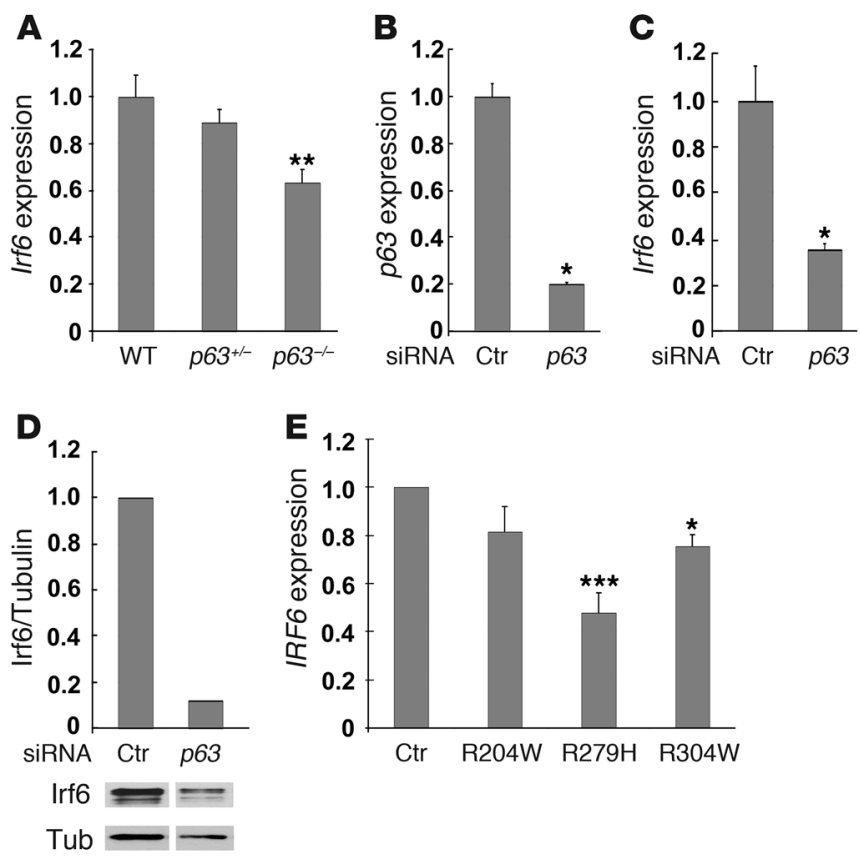

those detected in cell lines established from control individuals, and the presence of the early differentiation markers K1 and K10 suggested that differentiation had occurred normally at this stage (data not shown). Interestingly, all 3 cell lines exhibited reduced levels of IRF6 transcript compared with cells established from control individuals, and the decrease was statistically significant in cell lines R279H and R304W ( $P=0.001$ and 0.05 , respectively; Kruskal-Wallis 1-way ANOVA followed by post-hoc Dunn's test; Figure 4E). These data indicate that the correct function of $p 63$ is important for appropriate expression of IRF6.

IRF6 is activated by p63 through an enhancer element. To understand how IRF6 is regulated, we tested whether IRF6 is a direct transcriptional target of p63. ChIP in combination with deep sequencing analysis (ChIP-seq analysis) using chromatin isolated from a normal human primary keratinocyte cell line and a panp63 antibody identified a single p63 binding site within a 100-kb genomic region encompassing the IRF6 locus. This site was located approximately $10 \mathrm{~kb}$ upstream of the transcription start site of IRF6 and was a double peak with strong p63 binding affinity, as indicated by the number of sequenced tags (peak heights 229 and 58; Figure 5A). Peak detection using the model-based analysis of ChIP-seq (MACS) data program (28) revealed that the binding site from the double peak covered approximately 900 bp. Interestingly, peak 229 of this p63 binding site overlapped an enhancer element of IRF6 recently identified by searching for multispecies conserved sequences (Supplemental Figure 3), and a SNP in this enhancer has previously been associated with higher risk of cleft lip (13). This binding site was located within a broader site identified previously in a genome-wide study of p63 using ChIP-on-chip analysis of a carcinoma cell line (19), in which a second binding site was detected in intron 1 of IRF6 that was not detected in our ChIPseq analysis. The binding profile of $\mathrm{p} 63$ in close proximity to IRF6 was confirmed by independent ChIP-seq analysis using a second primary keratinocyte cell line established from a different normal individual (data not shown). The algorithm p53scan, which is suitable for binding motif searches for p53 family members (29), iden-

\section{Figure 4}

Irf6 in p63-deficient cells. (A) qPCR analysis of palatal shelves dissected from E13.5 wild-type, $p 63^{+/-}$, and $p 63^{-/-}$embryos indicated that Irf6 transcripts were reduced to approximately $90 \%$ and $63 \%$, respectively, of normal levels. ${ }^{* *} P=0.01$ versus $p 63^{+/-}$and wild-type (Mann-Whitney $U$ test). (B) siRNA knockdown of $p 63$ in mouse primary keratinocytes reduced $p 63$ levels 5 -fold. ${ }^{*} P=0.05$ versus control scrambled siRNA (Mann-Whitney U test). (C) Irf6 transcript levels after p63 siRNA knockdown were reduced more than $60 \%$. ${ }^{*} P=0.05$ versus control scrambled siRNA (Mann-Whitney U test). (D) Western analysis reveals reduced Irf6 protein levels in mouse primary keratinocytes after p63 siRNA knockdown. Quantitation, shown above the Western blot, shows Irf6 levels relative to tubulin. Samples were run on the same gel but were noncontiguous (white line). (E) qPCR analysis of IRF6 in human primary keratinocytes derived from normal control individuals and patients with EEC syndrome (R204W, R279H, and R304W) showed reduced IRF6 levels when the DNA-binding function of p63 was impaired. ${ }^{*} P=0.05,{ }^{* * *} P=0.001$ versus control (Kruskal-Wallis 1-way ANOVA followed by post-hoc Dunn's test). Data represent mean \pm SEM.

tified 2 consensus binding motifs in peak 229 and 1 additional motif in peak 58 (Figure 5B and Supplemental Figure 3).

To confirm the $\mathrm{p} 63$ binding site identified in ChIP-seq analysis, we performed independent ChIP-qPCR experiments using 2 p63 antibodies that recognize different epitopes in the p63 protein. Compared with 2 known targets of p63, the $p 21$ and BPAG genes (30, 31), ChIP efficiencies expressed as a percentage of input chromatin at peak 229 and peak 58 regions were higher than, or at least similar to, those of the known binding sites (Figure 5C). Use of 2 different p63 antibodies confirmed that the observed binding was specific for p63 (Figure 5C). Of note, the independent ChIP-qPCR analysis confirmed the lack of $\mathrm{p} 63$ binding to the previously reported site in the first intron of IRF6 (ref. 19 and Supplemental Figure $4 \mathrm{~A})$. As enhancer elements have been shown to exhibit tissue- and species-specific activities (32), we performed ChIP-qPCR in palatal shelves dissected from wild-type E13.5 mice. Consistent with our data from human primary keratinocytes, we observed p 63 binding to the upstream enhancer region of Irf6, but not to the intron 1 region in Irf6 (Supplemental Figure 4, B and C). We next assayed whether p63 binding is affected in patient keratinocyte cell lines with heterozygous $p 63$ mutations. For both peak 229 and peak 58 regions, we observed a marked reduction of $\mathrm{p} 63$ binding in keratinocytes with the EEC syndrome mutations R204W and R304W (Figure 5D). However, as both wild-type and mutant $\mathrm{p} 63$ proteins were present in the patient keratinocytes, it is probable that the detected residual DNA binding is mediated by the wild-type protein. To test this hypothesis further, we generated stable SAOS2-derived cell lines that express wild-type or $\Delta \mathrm{Np} 63 \alpha$ containing the mutation R304W in the absence of endogenous p63. ChIP experiments performed with these cell lines showed strong binding of wild-type p63 at the identified peak 58 binding site, whereas binding was abolished in the $\Delta \mathrm{Np} 63 \alpha \mathrm{R} 304 \mathrm{~W}$ cell line (Supplemental Figure 4D).

p63 can act as either an activator or a repressor, depending on the target gene. To investigate how the binding site regulates IRF6 transcription, we cloned the region surrounding peak 229 (Figure 5B) in a firefly luciferase reporter construct. In a transient transfection 


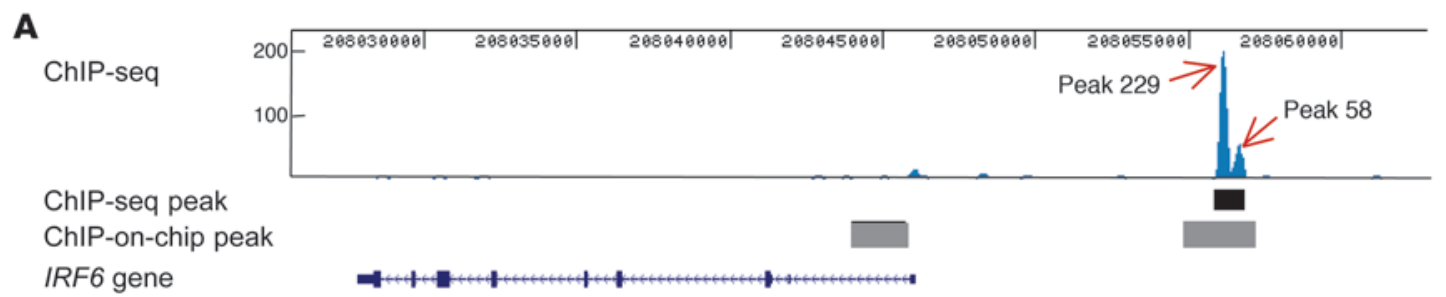

\section{B $\quad$ Motifs in peak 229}
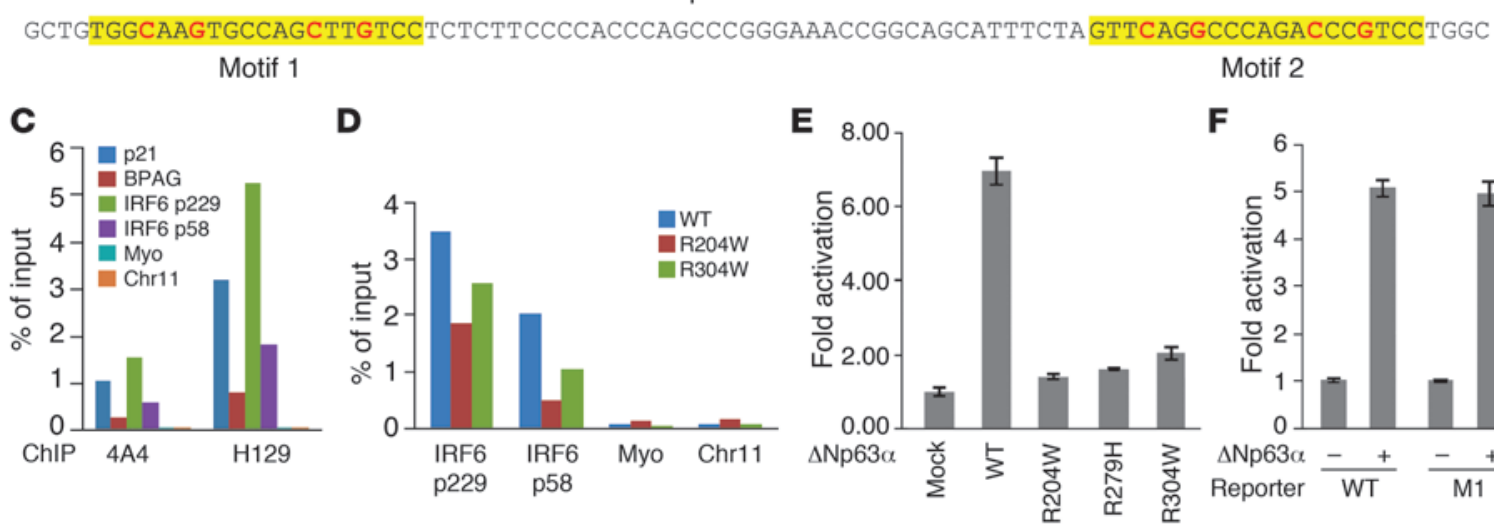

$\mathbf{F}$

Figure 5

A p63 binding site upstream of IRF6 functions as an enhancer element. (A) ChIP-seq analysis of p63 binding in human primary keratinocytes. The p63 binding site obtained from ChIP-seq is shown as a black bar below the double peak (peak 229 and peak 58 ), and p63 binding sites reported previously by ChIP-on-chip (19) are shown as gray bars. (B) The 2 p63 binding motifs identified within peak 229 are highlighted in yellow, with the most conserved cytosine and guanine bases shown in red. (C) ChIP-qPCR analysis of p63 binding using p63 antibodies 4A4 and H129. Specific binding of p63 to the positive controls p21 and BPAG as well as to peak 229 (p229) and peak 58 (p58) regions, but not to negative controls myoglobin exon 2 (myo) and a no-gene region (chr11), was observed. (D) ChIP-qPCR analysis of R204W and R304W cell lines indicated reduced p63 binding. (E) Transient transfection assays showed that wild-type p63 strongly activated transcription through peak 229. In contrast, activation by the p63 mutants R204W, R279H, and R304W was greatly reduced, with the highest level (approximately 2-fold) in R304W. (F) Site-directed mutagenesis of the conserved cytosine and guanine bases showed that both motifs in peak 229 were responsive to p63 and that mutation abolished transactivation. WT, reporter of wild-type peak 229; M1, mutation of motif 1; M2, mutation of motif 2; M1+M2, mutation of both motifs.

assay, wild-type $\Delta \mathrm{Np} 63 \alpha$ activated the luciferase reporter greater than 6-fold. In contrast, activation by the p63 mutants R204W, $\mathrm{R} 279 \mathrm{H}$, and R304W was greatly reduced (Figure 5E). These data are consistent with our results from the mouse model and from EEC syndrome patient keratinocytes in which IRF6 expression was dependent on functional p63 (Figure 3I and Figure 4, A and E). Furthermore, mutation analysis showed that both motifs identified in peak 229 were responsive to p63, and p63 transactivation was abolished when both motifs were mutated (Figure 5F). These data demonstrate that p63 binds directly to peak 229 to activate transcription. Taken together, our data establish that this binding site is an enhancer element through which p63 regulates expression of IRF6.

\section{Discussion}

Recent research has been highly successful in identifying the genetic mutations underlying syndromic forms of cleft lip and palate; for example, mutations in TBX22 cause X-linked cleft palate with ankyloglossia (33); in FOXE1, Bamforth-Lazarus syndrome (34, 35); in PVRL1, cleft lip and palate-ectodermal dysplasia syndrome (36); in MSX1, cleft lip and palate with tooth agenesis (37); in FLNA, otopalatodigital syndromes types 1 and 2 (38); in FGFR1, autosomal-dominant Kallmann syndrome (39); and in TFAP2A, branchio-oculo-facial syndrome (40). Moreover, variation within IRF6, TBX22, PVRL1, and $M S X 1$ is a contributing factor to nonsyndromic forms of cleft lip and cleft palate (41-46). Despite these successes, the molecular pathways in which the proteins encoded by these genes function during development of the lip and palate remain poorly characterized, as such studies are not feasible in human embryos. The identification and use of mouse models for orofacial clefting allows such networks to be characterized and the morphogenetic, cellular, and molecular changes involved in clefting to be dissected $(10,11,47)$.

In the current study, we used 2 different mutant mouse models to identify and characterize what we believe to be a novel interaction: mice heterozygous for both $p 63$ and the Irf6 knockin mutation R84C exhibited cleft palate. Although $\operatorname{Irf} 6^{+/ R 84 C}$ mice occasionally exhibited mild intraoral adhesions between the mandible and the maxilla that caused restricted opening of the mouth, failure to suckle, and neonatal lethality (14), neither of these mouse models displayed cleft palate in the heterozygous state, presumably because a critical threshold level for each of these genes during palate development in mice is reached. Importantly, we further demonstrated that IRF6 expression was dependent on functional $\Delta \mathrm{Np} 63 \alpha$ protein, which activated IRF6 transcription through an enhancer element located approximately $10 \mathrm{~kb}$ upstream of the transcription start site of IRF6. These results provide a molecular basis for the epistatic interaction between IRF6 and p 63 that is required for appropriate palatal development and thereby prevents cleft palate. Previous research has shown that $\mathrm{p} 63$ plays a central role in maintaining cellular prolifera- 
tion during development (48-50). For example, $\Delta \mathrm{Np} 63 \alpha$, the major p63 isoform expressed in primary keratinocytes and the palatal epithelia $(21,22,51)$, has been found to repress the transcription of numerous cell cycle mediators that act in an antiproliferative man-

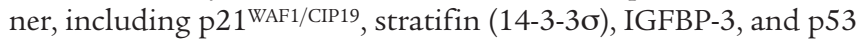
$(18,30,49,52)$. In addition to its role in maintaining proliferation, it has been proposed that $\mathrm{p} 63$ has an antiapoptotic role by negative regulation of transcription of proapoptotic molecules such as IGFIR and IGF-BP3 $(52,53)$; consequently, cell death is rarely observed in cells that overexpress $\Delta \mathrm{N}$-p $63 \alpha(18,54)$. In contrast, IRF6 promotes differentiation of mammary epithelial cells by promoting cell cycle arrest (55), and mice homozygous for either the R84C mutation or the loss-of-function mutation in Irf6 exhibit a hyperproliferative epidermis $(14,15)$. In wild-type mice, we observed high levels of Irf6 in the MEE of the palatal shelves, whereas p 63 was downregulated in the same cells as the palatal shelves approached each other. In contrast, in $\operatorname{Irf} 6^{R 84 C / R 84 C}$ mice, $\mathrm{p} 63$ expression was maintained throughout the MEE, which fail to degenerate and remain intact along the anteroposterior axis of the secondary palate (56). Taken together, these data suggest that downregulation of $\Delta \mathrm{Np} 63 \alpha$ in the MEE is a prerequisite for appropriate periderm development and palatal fusion and that persistent expression of $\mathrm{p} 63$ in $\operatorname{Irf6} 6^{R 84 C / R 84 C}$ mice prevents this process. Interestingly, consistent with our present data, Moretti and coworkers have demonstrated that induction of IRF6 by p 63 results in proteasome-mediated degradation of $\mathrm{p} 63$ and that the DNA-binding domain mutation R84C abolishes degradation of p63 (57). Previous research has shown that cytochalasin D inhibits periderm cell migration, resulting in failure of formation of the periderm-derived epithelial triangles, absence of periderm and basal MEE cell death, and incomplete adhesion of the palatal shelves (58). We therefore hypothesize that although the palatal shelves contact in $p 63^{+/-} I r f 6^{+/ R 84 C}$ embryos, the abnormal periderm cells that persist over the MEE at E15 act as a barrier to the formation of strong adhesion complexes between adjacent palatal shelves. As a consequence, the initial contact between the palatal shelves in

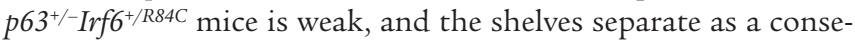
quence of continued craniofacial growth. In vitro culture experiments indicate that if contact is forced for a sufficient period of time, adhesion, and subsequent fusion of the palatal shelves, occurs. Together, observations from our $p 63^{+/-} \operatorname{Irf} 6^{+/ R 84 C}$ mouse model and possible feedback regulation of $\mathrm{p} 63$ by IRF 6 indicate that reciprocal regulation of $\mathrm{p} 63$ and IRF6 is essential for the appropriate specification of MEE fate during palatogenesis.

In previous biochemical studies, $\Delta \mathrm{Np} 63 \alpha$ has been shown to bind to many p53-responsive promoters and behave as a dominantnegative repressor toward p53 $(18,30,59)$. Recently, p63-specific target genes related to epithelial development have been reported, and $\Delta \mathrm{Np} 63 \alpha$ has also been shown to activate a subset of these genes (51); in contrast, no direct p63 target genes to our knowledge have been reported in the developing secondary palate. Here, we provide firm evidence that IRF6 is a direct target of p63 during palate development that can be activated by $\Delta \mathrm{Np} 63 \alpha$ through a p63-binding site upstream of the IRF6 gene. Interestingly, the binding site identified in this study overlaps with a recently reported regulatory element that was identified by searching for evolutionarily conserved sequences (13). The importance of this regulatory element during facial development is highlighted by its ability to drive reporter gene expression in the ectoderm covering the fusion sites between the developing facial prominences, consistent with known sites of endogenous Irf6 expression (13). As a SNP within this regulatory element has been shown to disrupt weak AP- $2 \alpha$ binding, owing to a nucleotide change in the degenerate AP- 2 motif, AP- $2 \alpha$ is thought to control IRF6 expression through this element (13). In the current study, we clearly show that p63 also strongly transactivates this element, raising the question of whether $\mathrm{p} 63$ and AP- $2 \alpha$ cooperate to regulate IRF6.

As cleft lip and cleft palate are not fully penetrant in patients with p63 mutations, genetic modifiers have been suggested to play a role in the disease pathway (7). Previous findings associating genetic variants in IRF6 with different cleft phenotypes (13), together with data obtained in the current study, lead us to speculate that IRF6 may act as a modifier in p63 disease phenotypes. Similarly, disease-causing IRF6 mutations have only been detected in approximately $70 \%$ of VWS families (60). Given that IRF6 can be regulated by the upstream $\mathrm{p} 63$ binding site, genetic variants in this site may disrupt normal transcriptional regulation of IRF6. Therefore, we believe the p 63 binding site identified in this study offers a new opportunity to search for the genetic causes of unresolved cases of nonsyndromic cleft lip and cleft palate as well as VWS and PPS that map genetically to the IRF6 locus, but in which the causative mutation has not been identified.

Our discovery of an interaction between 2 key transcription factors that contribute to cleft lip and cleft palate provides opportunities for further dissection of the pathways involved in the complex process of mammalian palate development. Further elucidation of how these genes are regulated and the identification of both shared and individual targets for $\mathrm{p} 63$ and IRF6 in future studies may unearth new candidates as contributing factors for the etiology of facial clefting.

\section{Methods}

Mouse breeding, genotyping, and phenotype analysis. Generation and genotyping of $p 63^{-/-}$and Irf6 $6^{R 84 / R 84 C}$ mice have been described previously $(14,22)$. $p 63$ knockout mice were provided by F. McKeon (Harvard Medical School, Boston, Massachusetts, USA), and Irf $6^{+/ R 84 C}$ mice were provided by R. Richardson (University of Manchester, Manchester, United Kingdom). Mice were housed in accredited animal facilities at the University of Manchester. All procedures were approved by the University of Manchester Ethical Review Committee and are licensed under the Animal (Scientific Procedures) Act 1986, issued by the Home Office. Compound heterozygous embryos were obtained by intercrossing $p 63^{+/-}$and $\operatorname{Irf} 6^{+/ R 84 C}$ mice, the morning of the vaginal plug being considered E0.5. Tissues dissected from E14.5 to P0 embryos were fixed in either $4 \%$ paraformaldehyde or Bouins reagent and processed for histological examination, scanning electron microscopy, or immunohistochemistry using standard protocols (22). Antibodies were obtained from P. Coulombe (K17; Johns Hopkins University, Baltimore, Maryland, USA); F. McKeon (p63, 4A4); D. Garrod (desmoplakin, 115F; University of Manchester, Manchester, United Kingdom); Covance Research Products (loricrin and K10); BD Biosciences (E-cadherin); Abcam ( $\alpha$ - and $\beta$-catenin), and R\&D Systems (activated caspase 3 ). The section in situ hybridization, cell proliferation assays, and transmission electron microscopy have been described previously $(14,22)$. For palate culture,

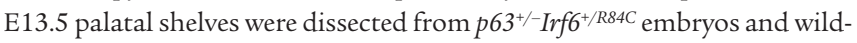
type littermates and cultured as described previously (61).

Cell culture. Patients with EEC syndrome caused by mutations in the $p 63$ gene were requested to donate a skin biopsy for research purposes. All procedures were approved by the ethical committee of the Radboud University Nijmegen Medical Centre ("Commissie Mensgebonden Onderzoek ArnhemNijmegen”). After informed consent was obtained, 3 punch biopsies ( $3 \mathrm{~mm}$ ) were taken from the lower back of each patient. Skin biopsy procedures, establishment of human primary keratinocytes from skin biopsies, and 
maintenance of primary keratinocyte cell lines were carried out as described previously (17). The Tet-on inducible expression system established in SAOS2 cells and described previously (29) was used to generate myc-tagged mouse $\Delta \mathrm{Np} 63 \alpha$ wild-type and R304W stable cell lines. Constructs for generation of SAOS2 stable cell lines were provided by M. Lohrum (Nijmegen Centre for Molecular Life Sciences, Radboud University Nijmegen, Nijmegen, The Netherlands). Doxycyclin (Sigma-Aldrich) was used to induce expression of myc- $\Delta \mathrm{Np} 63 \alpha$ wild-type and myc- $\Delta \mathrm{Np} 63 \alpha \mathrm{R} 304 \mathrm{~W}$ proteins at similar levels.

Constructs and transactivation assays. The genomic regions of $\mathrm{p} 63$ binding site peak 229 was amplified by PCR using gateway cloning primers (Supplemental Table 1) and cloned into a modified SmaI site in pGL3-Enhancer Vector, which contains a firefly luciferase reporter gene followed by a SV40 enhancer. To mutate the $\mathrm{p} 63$ binding motifs, the most conserved cytosine and guanine bases (Figure 5, red text) were mutated to adenosine. The $\Delta \mathrm{Np} 63 \alpha$ wild-type expression plasmid has been described previously (17). Point mutations were introduced into this plasmid to generate R204W, R279H, and R304W mutations. Transfection and luciferase assays were described previously (17).

RNA extraction and cDNA synthesis. RNA isolation was performed from 48-hour differentiated keratinocyte cultures as described previously (17) and from mouse palatal tissue using the Qiagen RNeasy kit. Total RNA was transcribed into cDNA according to the manufacturer's protocol using reverse transcriptase PCR iScript cDNA Synthesis kit (BioRad) and random primers in combination with MMLV reverse transcriptase (Invitrogen). The cDNA produced was purified by NucleoSpin Extract II kit (MachereyNagel, Bioke) according to the manufacturer's protocol.

ChIP and ChIP-seq. Primary keratinocytes and SAOS- 2 cultures were crosslinked with $1 \%$ formaldehyde for 10 minutes, and chromatin was collected as described previously (62). Chromatin was sonicated using a Bioruptor sonicator (Diagenode) 2 times, each for 8 minutes at high power, 30 seconds on, 30 seconds off. Antibodies 4A4 (Abcam) and H129 (Santa Cruz) were used in ChIP-qPCR experiments, as they are raised against the N-terminus (aa 1-205) and the $\alpha$-tail of p63 (aa 513-641), respectively. The binding of these antibodies was therefore predicted to be unaffected by DNA-binding domain mutations. Antibody 4A4 was used for ChIP-seq analysis. ChIP experiments were performed as previously described (29). ChIP-seq analysis was performed on a Solexa sequencing machine (Illumina) as described previously (63). All 32-bp sequence reads were uniquely mapped to the human genome NCBI build 36.1 (hg18) with 0 or 1 mismatch using ELAND (Illumina), which gave 3.2 million unique reads. Peak recognition was performed using MACS (28) with default settings and a $P$ value threshold of $1 \times 10^{-9}$.

siRNA transfection. Primary mouse keratinocytes were cultured in medium at low calcium concentrations and transfected with $200 \mathrm{nM}$ of validated siRNAs for mouse $\triangle \mathrm{N}$-p $63 \alpha$ mRNA (Invitrogen SiDNp63a-732 siRNA; sense, UCACAACAGUCCUGUACAAUUUCAU; antisense, AUGAAAUUGUACAGGACUGUUGUGA) and corresponding control scrambled siRNAs as previously described (64). Cells were analyzed 48 hours after transfection by real-time RT-PCR.

qPCR. qPCR primers were designed using Primer 3 (http://frodo.wi.mit. edu), and qPCR reactions were performed in a 7500 Fast Real Time PCR System apparatus (Applied Biosystems) using iQ SYBR Green Supermix (BioRad) according to the manufacturer's protocol. For qPCR of cDNA analysis, 3 exon-spanning primer sets for IRF6 were used (Supplemental Table 2). Human acidic ribosomal protein was used as a housekeeping gene to normalize the amount of cDNA. Differences in relative IRF6 expression between wild-type and mutant samples were calculated by the $2^{\Delta \Delta \mathrm{Ct}}$ method $(65,66)$. The relative expression was averaged using 3 primer sets. For
qPCR of ChIP analysis, 1 primer set was used for each tested binding region (Supplemental Table 3), and ChIP efficiency of certain binding sites was calculated using percentage of ChIPped DNA against input chromatin. Occupancy used in ChIP experiment with SAOS2 cells was calculated using ChIP efficiency of IRF6 P58 region standardized by that of myoglobin exon 2 region. For qPCR of palatal cDNA, mouse Gapdh (4352932E) and mouse Irf6 (Mm00516797_m1) TaqMan probes (Applied Biosystems) were used. 20- $\mu 1$ reactions were established in $1 \times$ PCR buffer (Invitrogen) containing $5 \mathrm{U}$ Taq polymerase (Invitrogen), $100 \mathrm{mM} \mathrm{MgCl}_{2}, 4 \mathrm{mM} \mathrm{dNTP}$, and $4 \mu \mathrm{l}$ cDNA. Reactions were performed in triplicate on an OpticonII thermal cycler (MJ Research). Relative levels of expression were calculated from a standard curve and normalized to Gapdh.

Bioimaging. Immunofluorescence and histology images were acquired on a Leica DMRB and DMLB, respectively, using $\times 2.5$ to $\times 63$ Plan Fluotar lenses and a SPOT RT camera (Diagnostic Instruments). For deconvolution, images were acquired on a Delta Vision RT (Applied Precision) restoration microscope using $\times 40 / 1.3$ Uplan FLN and $\times 60 / 1.42$ Plan Apo objectives and the Sedat filter set (Chroma 89000). The images were collected using a Coolsnap HQ (Photometrics) camera with a $\mathrm{Z}$ optical spacing of $0.2 \mu \mathrm{m}$. Raw images were deconvolved using Softworx software, and single optical sections of a deconvolved stack are shown.

Statistics. Mann-Whitney $U$ statistical tests were performed for Figure 4, A-C, using SPSS software. Kruskal-Wallis 1-way ANOVA followed by posthoc Dunn's test was performed for Figure 4E, using Prism software. A $P$ value less than 0.05 was considered significant.

\section{Acknowledgments}

We thank Les Lockey and Samantha Newby (University of Manchester Electron Microscopy Unit) for help and advice, Jane Kott and Robert Fernandez for help with the microscopy, and Marion Lohrum for assistance with Solexa sequencing analysis. The Bioimaging Facility microscopes used in this study were purchased with grants from BBSRC, Wellcome, and the University of Manchester Strategic Fund. This research was funded by grants from the Medical Research Council (G0400264 to J. Dixon and G0400955 to M.J. Dixon), Wellcome Trust (082868 to M.J. Dixon), the NIH (P50-DE016215 to M.J.D and AR39190 to G.-P. Dotto), the Healing Foundation (to M.J. Dixon), and EU (EPISTEM FP6-2004LIFESCIHEALTH-5, Integrated Project LSH-1.2.1-3 to H. von Bokhoven, H. Zhou, and G.-P. Dotto; Sixth Framework Program LSHB-CT-2005-019067 to G.-P. Dotto). M.J. Dixon is supported by the NIHR Manchester Biomedical Research Centre.

Received for publication June 22, 2009, and accepted in revised form February 24, 2010.

Address correspondence to: Jill Dixon, Faculty of Medical and Human Sciences, Manchester Academic Health Sciences Centre, Michael Smith Building, University of Manchester, Oxford Road, Manchester M13 9PT, United Kingdom. Phone: 44.161.275.5217; Fax: 44.161.275.5082; E-mail: jill.dixon@manchester.ac.uk. Or to: Hans van Bokhoven, Department of Human Genetics, Nijmegen Centre for Molecular Life Sciences, Radboud University Nijmegen Medical Centrum, Geert Grooteplein Zuid 10, 6525 GA Nijmegen, The Netherlands. Phone: 31.24.3616696; Fax: 31.24.3668752; E-mail: H.vanBokhoven@antrg.umcn.nl.
1. Vanderas AP. Incidence of cleft lip, cleft palate, and cleft lip and palate among races: a review. Cleft Palate J. 1987;24(3):216-225.

2. Murray JC, et al. Clinical and epidemiologic studies of cleft lip and palate in the Philippines. Cleft Palate Craniofac J. 1997;34(1):7-10.

3. Murray JC. Gene/environment causes of cleft lip and/or palate. Clin Genet. 2002;61(4):248-256.
4. Mossey PA, Little J, Munger RG, Dixon MJ, Shaw WC. Cleft lip and palate. Lancet. 2009; 374(9703):1773-1785.

5. Jones MC. Etiology of facial clefts: prospective evalua- 
tion of 428 patients. Cleft Palate J. 1988;25(1):16-20.

6. Celli J, et al. Heterozygous germline mutations in the p53 homolog p63 are the cause of EEC syndrome. Cell. 1999;99(2):143-153.

7. van Bokhoven H, Brunner HG. Splitting p63. Am J Hum Genet. 2002;71(1):1-13.

8. Kondo S, et al. Mutations in IRF6 cause Van der Woude and popliteal pterygium syndromes. Nat Genet. 2002;32(2):285-289.

9. de Lima RL, et al. Prevalence and nonrandom distribution of exonic mutations in interferon regulatory factor 6 in 307 families with Van der Woude syndrome and 37 families with popliteal pterygium syndrome. Genet Med. 2009;11(4):241-247.

10. Gritli-Linde A. Molecular control of secondary palate development. Dev Biol. 2007;301(2):309-326.

11. Gritli-Linde A. The etiopathogenesis of cleft lip and cleft palate: usefulness and caveats of mouse models. Curr Top Dev Biol. 2008;84:37-138.

12. Taniguchi T, Ogasawara K, Takaoka A, Tanaka N. IRF family of transcription factors as regulators of host defense. Annu Rev Immunol. 2001;19:623-655.

13. Rahimov F, et al. Disruption of an AP- $2 \alpha$ binding site in an IRF6 enhancer is associated with cleft lip. Nat Genet. 2008;40(11):1341-1347.

14. Richardson RJ, et al. IRF6 is a key determinant of the keratinocyte proliferation/differentiation switch. Nat Genet. 2006;38(11):1329-1334.

15. Ingraham CR, et al. Abnormal skin, limb and craniofacial morphogenesis in mice deficient for interferon regulatory factor $6(\operatorname{Irf6})$. Nat Genet. 2006; 38(11):1335-1340.

16. Little HJ, et al. Missense mutations that cause Van der Woude syndrome and popliteal terygium syndrome affect the DNA-binding and transcriptional activation functions of IRF6. Hum Mol Genet. 2009; 18(3):535-545.

17. Rinne $\mathrm{T}$, et al. A novel translation re-initiation mechanism for the p63 gene revealed by aminoterminal truncating mutations in Rapp-Hodgkin/ Hay-Wells-like syndromes. Hum Mol Genet. 2008 17(13):1968-1977.

18. Yang A, et al. p63, a p53 homolog at 3q27-29, encodes multiple products with transactivating, death-inducing, and dominant-negative activities. Mol Cell. 1998;2(3):305-316.

19. Yang A, et al. Relationships between p63 binding, DNA sequence, transcription activity, and biological function in human cells. Mol Cell. 2006; 24(4):593-602.

20. Ghioni P, Bolognese F, Duijf PH, Van Bokhoven H, Mantovani R, Guerrini L. Complex transcriptional effects of p63 isoforms: identification of novel activation and repression domains. Mol Cell Biol. 2002;22(24):8659-8668.

21. Laurikkala J, Mikkola ML, James M, Tummers M, Mills AA, Thesleff I. p63 regulates multiple signaling pathways required for ectodermal organogenesis and differentiation. Development. 2006; 133(8):1553-1563.

22. Thomason HA, Dixon MJ, Dixon J. Facial clefting in Tp63 deficient mice results from altered Bmp4, Fgf8 and Shh signaling. Dev Biol. 2008;321(1):273-282.

23. Yang A, et al. p63 is essential for regenerative proliferation in limb, craniofacial and epithelial development. Nature. 1999;398 (6729):714-718.

24. Mills AA, Zheng B, Wang XJ, Vogel H, Roop DR, Bradley A. p63 is a p53 homologue required for limb and epidermal morphogenesis. Nature. 1999; 398(6729):708-713.

25. Wolff S, Talos F, Palacios G, Beyer U, Dobbelstein M, Moll UM. The $\alpha / \beta$ carboxy-terminal domains of $\mathrm{p} 63$ are required for skin and limb development. New insights from the Brdm2 mouse which is not a complete p63 knockout but expresses p63 $\gamma$-like proteins. Cell Death Differ. 2009;16(8):1108-1117.
26. Leigh IM, Navsaria H, Purkis PE, McKay IA, Bowden PE, Riddle PN. Keratins (K16 and K17) as markers of keratinocyte hyperproliferation in psoriasis in vivo and in vitro. BrJ Dermatol. 1995;133(4):501-511.

27. Greene RM, Pratt RM. Developmental aspects of secondary palate formation. J Embryol Exp Morph. 1976;36(2):225-245.

28. Zhang Y, et al. Model-based analysis of ChIP-Seq (MACS). Genome Biol. 2008;9(9):R137.

29. Smeenk L, et al. Characterization of genome-wide p53-binding sites upon stress response. Nucleic Acids Res. 2008;36(11):3639-3654

30. Westfall MD, Mays DJ, Sniezek JC, Pietenpol JA. The Delta Np63 alpha phosphoprotein binds the p21 and 14-3-3 sigma promoters in vivo and has transcriptional repressor activity that is reduced by Hay-Wells syndrome-derived mutations. Mol Cell Biol. 2003;23(7):2264-2276.

31. Osada M, et al. p63-specific activation of the BPAG1e promoter. J Invest Dermatol. 2005;125(1):52-60.

32. Visel A, et al. ChIP-seq accurately predicts tissue-specific activity of enhancers. Nature. 2009; 457(7231):854-858.

33. Braybrook C, et al. The T-box transcription factor gene TBX22 is mutated in X-linked cleft palate and ankyloglossia. Nat Genet. 2001;29(2):179-183.

34. Clifton-Bligh RJ, et al. Mutation of the gene encoding human TTF-2 associated with thyroid agenesis, cleft palate and choanal atresia. Nat Genet. 1998; 19(4):399-401.

35. Castanet $\mathrm{M}$, et al. A novel loss-of-function mutation in TTF-2 is associated with congenital hypothyroidism, thyroid agenesis and cleft palate. Hum Mol Genet. 2002;11(17):2051-2059.

36. Suzuki K, et al. Mutations of PVRL1, encoding a cellcell adhesion molecule/herpesvirus receptor, in cleft lip/palate-ectodermal dysplasia. Nat Genet. 2000; 25(4):427-430.

37. van den Boogaard MJ, Dorland M, Beemer FA, van Amstel HK. MSX1 mutation is associated with orofacial clefting and tooth agenesis in humans. Nat Genet. 2000;24(4):342-343.

38. Robertson SP, et al. Localized mutations in the gene encoding the cytoskeletal protein filamin A cause diverse malformations in humans. Nat Genet. 2003; 33(4):487-491.

39. Dode C, et al. Loss-of-function mutations in FGFR1 cause autosomal dominant Kallmann syndrome. Nat Genet. 2003;33(4):463-465.

40. Milunsky JM, et al. TFAP2A mutations result in branchio-oculo-facial syndrome. Am J Hum Genet. 2008;82(5):1171-1177.

41. Lidral AC, et al. Association of MSX1 and TGFB3 with non-syndromic clefting in humans. Am J Hum Genet. 1998;63(2):557-568.

42. Sozen MA, Suzuki K, Tolarova MM, Bustos T, Fernandez Iglesias JE, Spritz RA. Mutation of PVRL1 is associated with sporadic, non-syndromic cleft lip/palate in northern Venezuela. Nat Genet. 2001; 29(2):141-142.

43. Jezewski PA, et al. Complete sequencing shows a role for MSX1 in non-syndromic cleft lip and palate. J Med Genet. 2003;40(6):399-407.

44. Vieira AR, Orioli IM, Castilla EE, Cooper ME, Marazita ML, Murray JC. MSX1 and TGFB3 contribute to clefting in South America. J Dent Res. 2003; 82(4):289-292.

45. Suzuki Y, et al. In a Vietnamese population, MSX1 variants contribute to cleft lip and palate. Genet Med. 2004;6(3):117-125.

46. Avila JR, et al. PVRL1 variants contribute to nonsyndromic cleft lip and palate in multiple populations. Am J Med Genet A. 2006;140(23):2562-2570.

47. Juriloff DM, Harris MJ. Mouse genetic models of cleft lip with or without cleft palate. Birth Defects Res A Clin Mol Teratol. 2008;82(2):63-77.
48. Bakkers J, Hild M, Kramer C, Furutani-Seiki M, Hammerschmidt M. Zebrafish DeltaNp63 is a direct target of Bmp signaling and encodes a transcriptional repressor blocking neural specification in the ventral ectoderm. Dev Cell. 2002;2(5):617-627.

49. Lee H, Kimelman D. A dominant-negative form of $\mathrm{p} 63$ is required for epidermal proliferation in zebrafish. Dev Cell. 2002;2(5):607-616.

50. Keyes WM, Wu Y, Vogel H, Guo X, Lowe SW, Mills AA. p63 deficiency activates a program of cellular senescence and leads to accelerated aging. Genes Dev. 2005;19(17):1986-1999.

51. Romano RA, Ortt K, Birkaya B, Smalley K, Sinha S. An active role of the $\Delta \mathrm{N}$ isoform of $\mathrm{p} 63$ in regulating basal keratin genes $\mathrm{K} 5$ and $\mathrm{K} 14$ and directing epidermal cell fate. PLoS One. 2009;4(5):e5623.

52. Barbieri CE, Perez CA, Johnson KN, Ely KA, Billheimer D, Pietenpol JA. IGFBP-3 is a direct target of transcriptional regulation by DeltaNp63alpha in squamous epithelium. Cancer Res. 2005; 65(6):2314-2320.

53. Nahor I, Abramovitch S, Engeland K, Werner H. The p53-family members $\mathrm{p} 63$ and $\mathrm{p} 73$ inhibit insulin-like growth factor-I receptor gene expression in colon cancer cells. Growth Horm IGF Res. 2005; 15(6):388-396.

54. Dietz S, Rother K, Bamberger C, Schmale H, Mossner J, Engeland K. Differential regulation of transcription and induction of programmed cell death by human p53-family members $\mathrm{p} 63$ and $\mathrm{p} 73$. FEBS Letts. 2002;525(1-3):93-99.

55. Bailey CM, Abbott DE, Margaryan NV, KhalkhaliEllis Z, Hendrix MJ. Interferon regulatory factor 6 promotes cell cycle arrest and is regulated by the proteasome in a cell cycle-dependent manner. $\mathrm{Mol}$ Cell Biol. 2008;28(7):2235-2243.

56. Richardson RJ, Dixon J, Jiang R, Dixon MJ. Integration of IRF6 and Jagged 2 signalling is essential for controlling palatal adhesion and fusion competence. Hum Mol Genet. 2009;18(14):2632-2642.

57. Moretti F, et al. A regulatory feedback loop involving p63 and IRF6 links the pathogenesis of 2 genetically different human ectodermal dysplasias. J Clin Invest. 2010;120(5):1570-1577.

58. Cuervo R, Covarrubias L. Death is the major fate of medial edge epithelial cells and the cause of basal lamina degradation during palatogenesis. Development. 2004;131(1):15-24.

59. Ihrie RA, et al. Perp is a p63-regulated gene essential for epithelial integrity. Cell. 2005;120(6):843-856.

60. de Lima RL, et al. Prevalence and nonrandom distribution of exonic mutations in interferon regulatory factor 6 in 307 families with Van der Woude syndrome and 37 families with popliteal pterygium syndrome. Genet Med. 2009;11(4):241-247.

61. Ferguson MW, Honig LS, Slavkin HC. Differentiation of cultured palatal shelves from alligator, chick, and mouse embryos. Anat Rec. 1984;209(2):231-249.

62. Denissov $\mathrm{S}$, et al. Identification of novel functional TBP-binding sites and general factor repertoires. EMBO J. 2007;26(4):944-954.

63. Nielsen R, et al. Genome-wide profiling of PPARgamma:RXR and RNA polymerase II occupancy reveals temporal activation of distinct metabolic pathways and changes in RXR dimer composition during adipogenesis. Genes Dev. 2008;22(21):2953-2967.

64. Nguyen BC, et al. Cross-regulation between Notch and p63 in keratinocyte commitment to differentiation. Genes Dev. 2006;20(8):1028-1042.

65. Livak KJ, Schmittgen TD. Analysis of relative gene expression data using real-time quantitative PCR and the 2(-Delta Delta C(T) Method. Methods. 2001;25(4):402-408.

66. Pfaffl MW. A new mathematical model for relative quantification in real-time RT-PCR. Nucleic Acids Res. 2001;29(9):e45. 